\title{
Differences in Effectiveness among Devices for Endovascular Thrombectomy in Patients with Acute Ischemic Stroke
}

\author{
Marco Chiumente, ${ }^{\mathrm{a}}$ Sabrina Trippoli, ${ }^{\mathrm{b}}$ Andrea Messori ${ }^{\mathrm{b}}$ \\ altalian Society for Clinical Pharmacy and Therapeutics, Milano, Italy \\ ${ }^{b}$ HTA Unit, ESTAV Toscana Centro, Regional Health Service, Firenze, Italy
}

\section{Dear Sir:}

In the treatment of acute ischemic stroke, the proofs of effectiveness for endovascular thrombectomy have become more robust in recent times, but some questions remain on whether the effectiveness differs across different devices (e.g. Trevo, Merci, and Solitaire). In the absence of studies that have directly compared these devices with one another, comparative information relies on the results of meta-analyses, and particularly on indirect meta-analytic comparisons conducted with a network design.

In 2013, we published the first network meta-analysis in this field ${ }^{1}$ in which we evaluated three devices (Merci, Trevo, and Solitaire) based on pivotal randomized trials. Both Trevo and Solitaire were found to be more effective than Merci, but the information was not sufficient to determine if Solitaire was more effective also than Trevo.

Between 2013 and 2015, numerous randomized controlled trials (RCTs) evaluating these devices have been made available. Three "traditional" pairwise meta-analyses, that synthesized the effectiveness data resulting from direct comparisons, have been published in the Journal of Stroke, ${ }_{1}^{2} \mathrm{AAMA}_{1}^{3}$ and Journal of American College of Cardiologists, ${ }^{4}$ but no network meta-analysis based on the more mature evidence currently available has been conducted.

The meta-analysis by Hong et al. ${ }^{2}$ (15 trials) found that endovascular recanalization therapy was associated with increased good outcomes (0-2 modified Ranking Scale) compared to the controls given standard therapy (including intravenous tissue plasminogen activator in 9 trials); the odds ratio (OR) was 1.79 (95\% confidence interval, 1.34 to 2.40 ). Likewise, the meta-analysis by Badhiwala et al. ${ }^{3}$ (8 RCTs) found that, as compared with thrombolysis, the endovascular interventions increased the rate of functional independence at 90 days (0-2 modified Ranking Scale) with an OR of 1.71 (95\% confidence interval: 1.18 to 2.49). Also the meta-analysis by Elgendy et al. ${ }^{4}$ investigated the trials comparing thrombectomy with standard thrombolysis (8 $\mathrm{RCTs})$, and its results were virtually identical to those reported by Badhiwala et al. ${ }^{3}$

However, no indirect comparisons between individual devices were made in these three meta-analyses even though, for practical reasons, this information can be of great interest.

In this report, we describe one such network meta-analysis with indirect comparisons focused on these devices. Our description of these results was limited to a re-analysis of the standard pairwise meta-analysis data published by Badhiwala et al. ${ }^{3}$ (see Table 1 of Reference 3 ; total number of patients $=2,387$ ), which we re-evaluated by application of a Bayesian network metaanalysis. ${ }^{5}$ As in Badhiwala's study, our clinical material consisted of 8 RCTs comparing a stent-based endovascular intervention (treatment group) with thrombolysis (controls). Four of these trials did not employ, in the treatment group, a single device but three or more of these (typically: Penumbra or Trevo or Merci or Solitaire) while the remaining four trials employed Solitaire. The ESCAPE trial, in which Solitaire was employed in 66\% of the patients of the treatment group, was classified among the four studies employing Solitaire. Functional independence at 90 days 
Thromobolysis

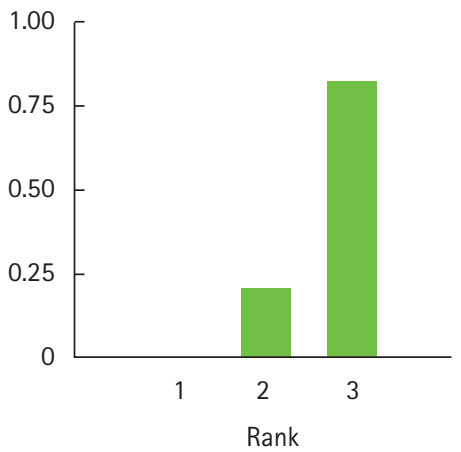

Any stent

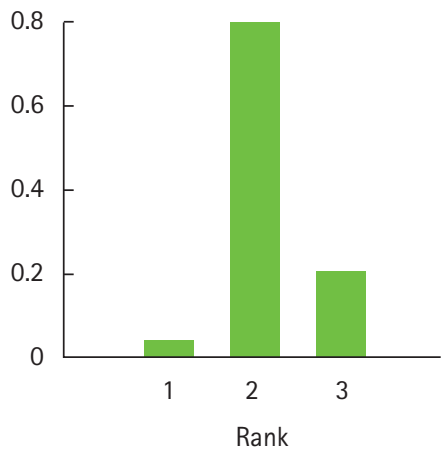

Solitaire

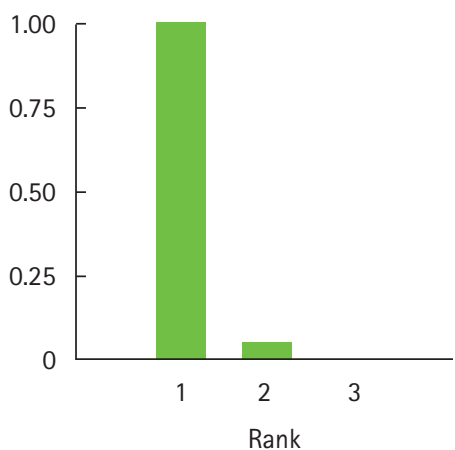

Figure 1. Comparative effectiveness of two endovascular interventions and thrombolysis evaluated according to Bayesian network meta-analysis (8 randomized studies; end-point = functional independence at 90 days). The figure shows the histogram of rankings estimated for each of the three treatments according to the Bayesian probabilistic analysis (random-effect model). Each panel indicates, in a series of simulations, how often the treatment concerned ranked first or second or third in effectiveness.

was our end-point. The OR, accompanied by its $2.5 \%$ to $97.5 \%$ credible interval $(\mathrm{Crl})$, was the outcome measure of our analysis. Since we anticipated some heterogeneity in the clinical material, our primary analysis used the random-effect model, but also the fixed-effect one was tested. ${ }^{5}$

The results of our Bayesian meta-analysis (random-effect model) showed that the patient group receiving any device had a numerical, but not significant higher incidence of functional independence than the controls $(O R=1.20 ; \mathrm{Crl}$ : 0.71 to 1.91). In contrast, the group receiving Solitaire had a significantly better incidence of this end-point as compared with the controls $(\mathrm{OR}=$ 2.52; Crl: 1.51 to 4.33). In the indirect comparison between Solitaire and any stent, the former had a significantly better effectiveness ( $\mathrm{OR}=2.11 ; \mathrm{Crl}: 1.06$ to 4.48$)$.

Figure 1 shows the ranking in effectiveness, an information that is typical of all Bayesian analyses; Solitaire ranked first (median rank $=1 ; \mathrm{Crl}: 1$ to 1 ) followed by any stent (median rank $=2$; $\mathrm{Crl}: 2$ to 3 ) and by thrombolysis (median rank $=3 ; \mathrm{Crl}: 2$ to 3 ). The results in terms of $\mathrm{OR}$ calculated according to the fixed effect model were quite similar (data not shown in detail) even though, as expected, the Crls were somewhat narrower. According to this model, the indirect comparison between Solitaire and any stent showed an OR of 2.07 (Crl: 1.43 to 2.97).

There was a significant degree of heterogeneity in the clinical material (8 RCTs) included in the meta-analysis by Badhiwala et al. ${ }^{3}$ this is generally considered a negative factor that limits the value of the evidence concerned. ${ }^{6}$ To study in more depth the degree of heterogeneity of this clinical material, in a separate analysis ${ }^{6}$ we selected the four RCTs that directly compared Solitaire with thrombolysis and we carried out a traditional pairwise meta-analysis. Interestingly enough, the pooled results from these 4 trials (end point: functional independence at 90 days) showed no heterogeneity with a significantly better effectiveness for the Solitaire group (pooled odds-ratio $=2.47 ; 95 \%$ confidence interval: 1.84 to $3.33 ; I^{2}=0 \%, P$ value for heterogeneity $\left.=0.689\right){ }^{6}$

In summary, the main advantages of our re-analyses of this clinical material include the evaluation of the most updated evidence on this topic and the use of a statistical technique that allows an indirect comparison between the devices. Our indirect comparisons indicate that Solitaire has a significantly better effectiveness than that of the other devices. However, owing to the well-known limitations of network meta-analyses ${ }^{5}$ and to the presence of heterogeneity in the overall series of 8 RCTs, our findings represent a hypothesis that needs further confirmation from other trials.

\section{References}

1. Messori A, Fadda V, Maratea D, Trippoli S. New endovascular devices for acute ischemic stroke: summarizing evidence by multiple treatment comparison meta-analysis. Ann Vasc Surg 2013;27:395-396.

2. Hong KS, Ko SB, Lee JS, Yu KH, Rha JH. Endovascular recanalization therapy in acute ischemic stroke: updated meta-analysis of randomized controlled trials. J Stroke 2015;17:268-281.

3. Badhiwala $\mathrm{JH}_{\text {, Nassiri }}$, Alhazzani $W$, Selim MH, Farrokhyar $F$, Spears J, et al. Endovascular thrombectomy for acute ischemic stroke: a meta-analysis. JAMA 2015;314:1832-1843.

4. Elgendy IY, Kumbhani DJ, Mahmoud A, Bhatt DL, Bavry AA. Mechanical thrombectomy for acute ischemic stroke: a metaanalysis of randomized trials. J Am Coll Cardiol 2015;66:24982505.

5. Higgins JP, Welton NJ. Network meta-analysis: a norm for comparative effectiveness? Lancet 2015;386:628-630. 
6. Messori A. Comment: meta-analysis on thrombectomy in acute ischemic stroke: is the degree of heterogeneity influenced by the type of device? PubMed Commons, published 24 December 2015, available at http://www.ncbi.nlm.nih.gov/pub med/26529161\#cm26529161_13432.
Correspondence: Marco Chiumente

Italian Society for Clinical Pharmacy and Therapeutics, via Farini 81, 20100 Milano, Italy

Tel: +39-3457021796, Fax: +39-02-6686699

E-mail:md3439@me.com

Received: November 19, 2015

Revised: December 25, 2015

Accepted: January 6, 2016

The authors have no financial conflicts of interest. 